\title{
XXXV. Ueber optische Erscheinungen am Quarz.
}

\author{
Von \\ C. Viola in Rom. \\ (Hierzu 1 Textfigur.)
}

Wenn eine Krystallfläche ausgezeichnet geschliffen wird, wie z. B. ein Quarzschnitt sich schleifen lässt, kann man mit dem Abbe'schen Apparate eine Genauigkeit von einigen Finheiten in der fünften Decimalstelle erreichen. Um dieses Resultat zu bekommen, ist es zweckmässig, eine kleine Papieroder Staniolblende zwischen Spiegel und Halbkugel einzuschieben. Ist das verwendete Licht stark und dic Blende recht klein (in der Art eines kleinen Schlitzes), so erscheint dic (Grenze der Totalreflexion ausgezeichnet scharf. Diese Methode habe ich sowohl in München bei meinen Feldspathstudien, als nachher in Rom angewendet.

In München arbcitete ich bei den leldspathsludien stets mit der Jifferentialmethode und dazu gebrauchte ich als Vergleichungszahl den Brechungsindex $\omega$ des Quarzes. Für diese Beobachtungen verfügte ich über zwei Schnitte parallel und senkrecht der IIauptaxe eines wasserhellen Quarzkrystalles. Nun zeigte es sich, dass zur leststellung des Grenzwinkels für $\omega$ die zwei um $180^{\circ}$ entfernten Ablesungen nicht genügten, da sie immer verschiedenes arithmetisches Mittel gaben. Ich war daher gezwungen, bei meinen Untersuchungen stets vier um $90^{\circ}$ entfernte Ablesungen zu machen, um daraus ein constantes arithmetisches Mittel zu erhalten. Da diese im Quarz beobachteten kleinen Differenzen nicht von den kleinen im Apparate steckenden Fehlern herrühren, gebe ich hier die an der Mikrometerschraube des $\Lambda$ bbe'schen Apparates gemachten Ablesungen von $0^{\circ}$ bis $360^{\circ}$ des Horizontalkreises, entsprechend der Grenzlinie der Totalreflexion für Luft und Glaskugel. 


\begin{tabular}{|c|c|c|}
\hline $\begin{array}{l}\text { Horizontal- } \\
\quad \text { kreis : }\end{array}$ & $\begin{array}{l}\text { Mikrometer- } \\
\text { schraube: }\end{array}$ & $\begin{array}{l}\text { Arithmetisches Nittel } \\
\text { von zwei um } 180^{\circ} \\
\text { ent[ernten } \Lambda \text { blesungen }\end{array}$ \\
\hline $0^{0}$ & $3^{\prime} 35^{\prime \prime}$ & $6^{\prime} 10^{\prime \prime}$ \\
\hline 20 & 520 & 65 \\
\hline 10 & 520 & (j) 5 \\
\hline 60 & ப̆ 30 & 610 \\
\hline 80 & 540 & $6 \xi$ \\
\hline 100 & 550 & $\xi$ \\
\hline 120 & 60 & 6 \\
\hline 140 & 620 & 6 \\
\hline 160 & 633 & 6 \\
\hline 180 & 650 & - \\
\hline 200 & 650 & - \\
\hline 220 & 650 & - \\
\hline 240 & 650 & - \\
\hline 260 & 630 & - \\
\hline 280 & 620 & - \\
\hline 300 & 555 & - \\
\hline 320 & 545 & 一 \\
\hline 340 & bै 1.0 & - \\
\hline
\end{tabular}

Man ist also im Stande sehr scharf einzustellen, und zwar liegt der Fehler zwischen $+10^{\prime \prime}$ und - $10^{\prime \prime}$.

Die kleinen Differenzen in den directen Ablesungen rühren offenbar von der nie wegzubringenden kleinen Neigung der Fläche der Halbkugel in Bezug auf die verticale Drehungsaxe her, Differenzen, die durch das arithmetische Mittel verschwinden.

Die kleine aus Stanniol gefertigte und zwischen Spiegel und Glaskugel angebrachte Blende hatte den $Z$ weck, die Beleuchtung auf eine sehr kleine am Centrum der Kugel liegende Stelle zu beschränken.

Ich lasse nun die nämlichen Beobachtungen für die Grenzlinien eines wasserhellen Quarzes folgen, von dem ich zwei Schnitte, d. h. parallel und senkrecht, zur dreizähligen $\Lambda x e$ zur Hand hattc.

Grenzwinkeldifferenzen für Quarz, parallel zur dreizähligen Axe geschnitten.

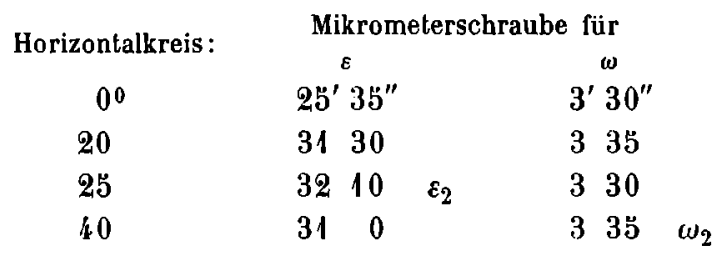




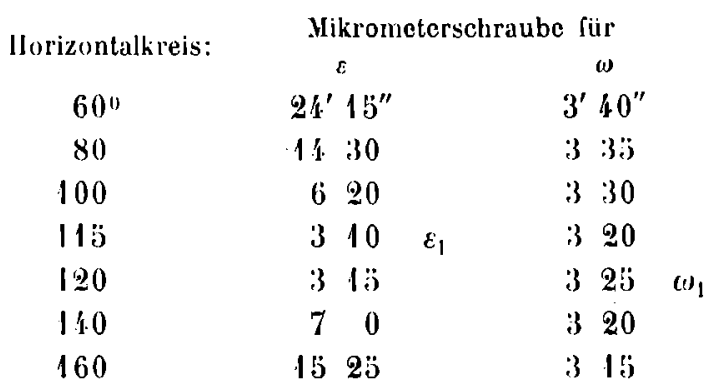

Die bei $w$ erhallenen arithmetischen Mittel sind nicht gleich. Der Unlerschied betrïgt $2: 3^{\prime \prime}$.

Die aus den direclen Beolbachlungen berechneten arilhmetischen Mittel folgen einer gewissen Regel, sowie diejenigen Zalılen, die dem Brechungsindex $\varepsilon$ entsprechen. Man ist also nicht berechligt anzunehmen, der Unterschied von $2 b^{\prime \prime}$ rühre von einem \%ufall her.

Bekanntlich können dje Fresnel'schen Geselze für Quarz und für jeden optisch cinaxigen Krystall so ausgedrückl werden: l)ie optische Strahlenfläche oder Wellenfliiche besteht aus cinem Rotationsellipsoid und einer Kugel, die sich in zwci gegenüberliegenden l'unkten berühren, durch welche die optische Ixe geht. Diese Fresnel'schen Gesetze gehen aus der ljndulationstheoric des Lichles unmittelbar hervor. Nach derselben müssen die bei $\omega$ heobachtelen Zahlen gileich sein, nder einen Fehler von $\pm 10^{\prime \prime}$, höchstens $\pm 15^{\prime \prime}$ zeigen, und zwar gleichmïssig in der Drehung des Horizontalkreises vertheilt sein.

Die gemachten Erfahr'ungen zeigen, dass dic l'resnel'schen Gesetze für Quarz nicht gelten. Dic Grenzcurve der Totalreflexion für $\omega$ des Quarzes ist in dem zur llauplaxe parallelen Schnitle nicht ein Kreis, wie es nach den Fresnel'schen Gesetzen scin sollte, sondern eine Ellipse. Wir haben es in den oben angegebenen zwei Colonnen mit zwei Maximis und mit zwei Minimis zu thun, welche wir mit $\varepsilon_{2}$ und $\omega_{2}$ resp. mit $\varepsilon_{1}$ und $\omega_{1}$ bezeichnen wollen.

Wir berechnen jetzt die Brechungsindices, denen diese beobachteten Zahlen entsprechen. Da hier nur Differenzen in lirage kommen, so gehen wir von einem gebräuchlichen Datum aus. Es sei also

$$
\varepsilon_{2}=1,55338 \text {. }
$$

Dann haben wir den entsprechenden Winkel der Totalreflexion

$$
\varphi_{\varepsilon_{2}}=55^{0} 15^{\prime} 26,5^{\prime \prime} \text {. }
$$

Da der Unterschied zwischen der ersten und zweiten Colonne bei $\varepsilon_{1}$ und $\omega_{1}$ nur $5 "$, höchstens $10^{\prime \prime}$ beträgt, so können wir die zwei entsprechenden Winkel gleich setzen, also eine Winkeldifferenz von $3^{\prime} 15^{\prime \prime}$ an- 
nehmen. Unter dieser Voraussetzung berechnet sich der Grenzwinkel für $\varepsilon_{1}$ und $\omega_{1}$ folgendermaassen:

also

$$
\begin{gathered}
55^{0} 15^{\prime} 26,5^{\prime \prime} \\
\varphi_{\omega_{1}}=\varphi_{\varepsilon_{1}}=\frac{-2855,0}{54^{0} 46^{\prime} 31,5^{\prime \prime}-} \\
\omega_{1}=\varepsilon_{1}=1,54426 .
\end{gathered}
$$

Ebenso berechnet sich der Grenzwinkel für $\omega_{2}$ wie folgt:

$$
\begin{gathered}
55^{0} 15^{\prime} 26,5^{\prime \prime} \\
-2830 \\
\varphi_{\omega_{2}}=54^{0} 46^{\prime} 56,5^{\prime \prime} \\
\omega_{2}=1,54440 \\
\omega_{2}-\omega_{1}=0,00014 .
\end{gathered}
$$

daher

und

Grenzwinkeldifferenz für Quarz, senkrecht zur dreizähligen Axe geschnitten.

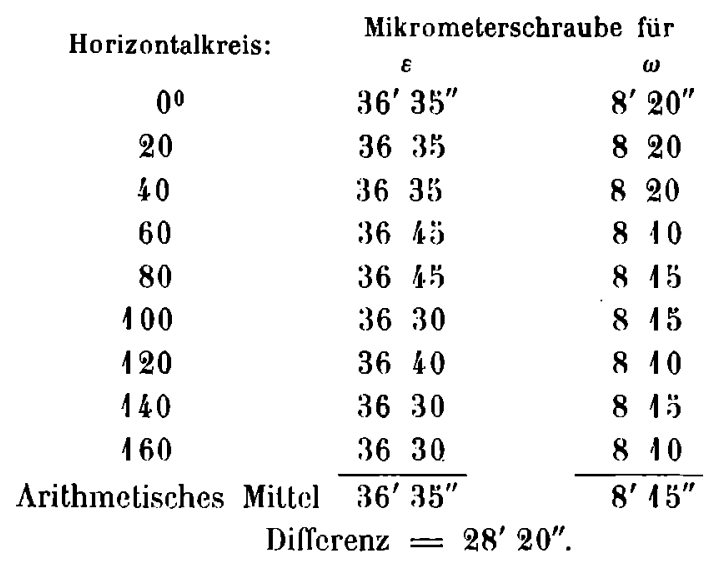

Die sowohl bei $\omega$ als auch bei $\varepsilon$ crhaltenen Winkeldifferenzen zeigen kleine Unterschiede, die 15" nicht überschreiten. Wir sehen daher, dass die Grenzcurven der Totalreflexion für einen Schnitt senkrecht zur dreizähligen $\Lambda$ xe des Quarzes zwei Kreise sind. Nehmen wir, wie früher, an :

$$
\varepsilon_{2}=1,55338 \text { und } \varphi_{\varepsilon_{2}}=\ddot{5} 5^{0} 13^{\prime} 26,3^{\prime \prime} \text {, }
$$

so berechnet sich

und daher

$$
\begin{aligned}
& \varphi_{\omega_{2}}=544^{0} 47^{\prime} 6,5^{\prime \prime} \\
& u_{2}=1,5444.3 .
\end{aligned}
$$

Vergleichen wir diese Zahl mit der früher erhaltenen und berechnen wir das arithmetische Mittel, so erhalten wir folgende Zusammenstellung:

$$
\begin{array}{ll}
\varepsilon_{1}=1,54426 & \omega_{1}=1,54426 \\
\varepsilon_{2}=1,55338 & \omega_{2}=1,54442
\end{array}
$$


Die Strahlenfläche oder Wellenfläche des Quarzes besteht in Folge dessen aus zwei Rotationsellipsoiden (siehe die Figur), die sich in den Scheitelpunkten berühren, durch welche die sogenannte optische Axe geht.

Die Abweichung der beim Quarz für die optischen Erscheinungen geltenden Gesetze von den Fresnel'schen ist nicht gross, aber sie ist vorhanden und muss erklärt werden. Natürlich dürfen die hierauf sich beziehenden Erklärungen der optischen Erscheinungen nicht von der Undulationstheorie des Lichtes ausgehen, denn diese sagt nicht mehr aus, als die Fresnelschen Gesetze.

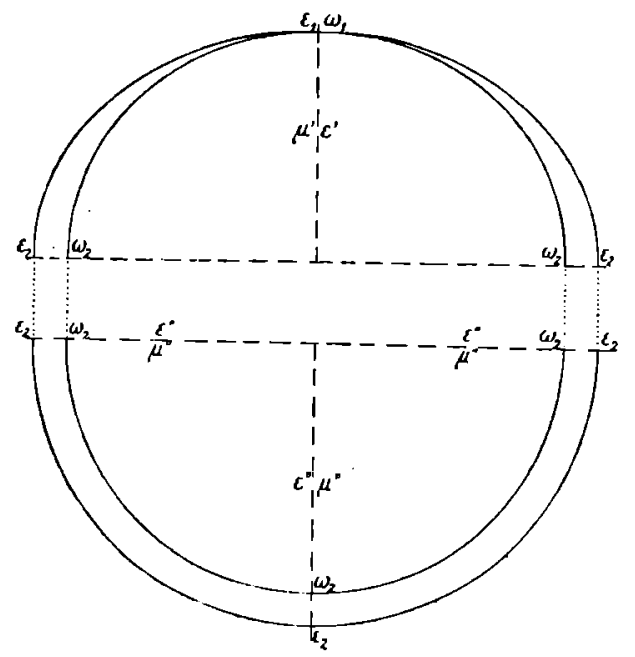

Die neue Theorie des Lichtes drückt aus, dass das Iicht in einer elektrischen Störung besteht, welche immer mit einer magnetischen Störung verbunden ist. Wir haben also bei dieser Auffassung sowohl die hauptdiëlektrischen Constanten, als auch die hauptmagnetischen Constanten in Betracht zu ziehen. Die aus dieser Theorie hervorgehenden Gleichungen für die Lichterscheinungen werden aus den Maxwell'schen Differentialgleichungen der Bewegung entwickelt, die später von Hertz verallgemeinert worden sind. Wir wollen sie aber nicht anführen, da sie schon längst einer eingehenden Betrachtung unterzogen worden sind ${ }^{1}$ ).

Bei dieser Gelegenheit beschränken wir uns darauf, nur die Gleichungen aufzuschreiben, welche diejenigen von Fresnel ersetzen müssen, und zwar bleiben wir speciell bei dem Falle des Quarzes. Diesen besonderen Fall hat Helmholtz ${ }^{2}$ ) in seiner Berliner Vorlesung im Jahre 1892 behandelt.

Die Geschwindigkeit $c$ des Lichtes wird durch folgende zwei Helmholtz'sche Gleichungen ausgedrückt:

$$
\begin{aligned}
& c_{1}{ }^{2}=\frac{1}{A^{2} \mu^{\prime}}\left[\frac{\cos ^{2} \alpha}{\varepsilon^{\prime}}+\frac{\sin ^{2} \alpha}{\varepsilon^{\prime \prime}}\right], \\
& c_{2}{ }^{2}=\frac{1}{A^{2} \varepsilon^{\prime}}\left[\frac{\cos ^{2} \alpha}{\mu^{\prime}}+\frac{\sin ^{2} \alpha}{\mu^{\prime \prime}}\right]
\end{aligned}
$$

1) A. Sella, Sulle leggi di propagazione della luce nei cristalli magnetici. R. Accad. d. Lincei, Roma 1895, 1, 1 .

2) Vergl. H. von Helmholtz, Vorlesungen über die elektromagnetische Theorie des Lichtes; herausgegeben von A. König und K. Runge. 1897, S. 355-363. 
wohei $A$ die das elektromagnetische Maass in elektrostatisches umwandelnde Constante und $\alpha$ den Winkel, den dic Normale der Wellenchene mit der dreizähligen $\Lambda$ xe bildet, bedeuten.

$$
\begin{aligned}
& \varepsilon^{\prime} \text { und } \varepsilon^{\prime \prime} \text { sind dic hauptdiëlektrischen Constanten, } \\
& \mu^{\prime}-\mu^{\prime \prime}-\text { - hauptmagnetischen - }
\end{aligned}
$$

des Quarzes.

Sowohl $c_{1}$ als $c_{2}$ ändern sich mit dem Winkel $\alpha$, also mit der Richtung des Vectors.

Die von Helmholtz gemachte $\Lambda$ nnahme, dass die lichtungen, nach welchen $\varepsilon^{\prime}$ und $\varepsilon^{\prime \prime}$ gemessen werden, mit denjenigen, nach welchen $\mu^{\prime}$ und $\iota^{\prime \prime}$ gemessen werden, zusammenfallen, ist der einfachste Fall und entspricht den Eigenschaften des Quarzes vollkommen.

Die elektromagnetische Theoric des Lichtes deckt sich mit der Undulationstheorie, wenn, wic es gewöhnlich geschieht, $\mu_{0}=\mu_{1}$ ist, denn dann ist $c_{2}=$ constans, wic die Fresnel'schen Gesetzc verlangen.

$\Lambda$ ndere Fälle werden bei Krystallen vorkommen, in denen die elektromagnelische Theorie des Lichtes ihre Bestätigung finden wird. Jedenfalls aber werden von zwei isomorphen Substanzen, dercn eine den Fresnelschen Gesetzen folgt, die andere nicht, die isomorphen Mischungen beider denselben nicht folgen dürfen. Jie in diesem Sinne gemachten Erfahrungen werden in die Kenntniss der isomorphen Mischungen viel Iicht bringen.

Was die clektromagnetischen Constanten des Quarzes anbelangt, so erhalten wir aus den gemachten Beobachlungen und den oben angegebenen Gleichungen :

$$
\begin{aligned}
\text { für } \alpha=0^{0} \quad \text { ist } \sin \alpha=0, \quad \cos \alpha=1, c_{1}=\frac{V}{\varepsilon_{1}}, c_{2}=\frac{V}{\omega_{1}}, \\
-\alpha=90^{\circ}-\quad-\quad=1-\quad=0 \quad c_{1}=\frac{V}{\varepsilon_{2}}, c_{2}=\frac{V}{\omega_{2}} .
\end{aligned}
$$

Daraus

$$
\omega_{1}^{2}=\varepsilon_{1}^{2}=\frac{A^{2}}{V^{2}} \mu^{\prime} \varepsilon^{\prime}, \quad \varepsilon_{2}^{2}=\frac{A^{2} \mu^{\prime} \varepsilon^{\prime \prime}}{V^{2}}, \quad \omega_{2}^{2}=\frac{A^{2} \mu^{\prime \prime} \varepsilon^{\prime}}{V^{2}} .
$$

In Folge dessen

$$
\frac{\varepsilon^{\prime \prime}}{\varepsilon^{\prime}}=\left(\frac{\varepsilon_{2}}{\varepsilon_{1}}\right)^{2}, \quad \frac{\mu^{\prime \prime}}{\mu^{\prime}}=\left(\frac{\omega_{2}}{\sigma_{1}}\right)^{2},
$$

somit ausgerechnet:

$$
\frac{\varepsilon^{\prime \prime}}{\varepsilon^{\prime}}=1,01185 ; \quad \frac{\mu^{\prime \prime}}{\mu^{\prime}}=1,00020 .
$$

